\title{
Hypoglycaemia in Type 2 Diabetes - Clinical Consequences and Impact on Treatment
}

\author{
Chantal Mathieu, ${ }^{1}$ Claudia Filozof ${ }^{2}$ and Anthony $\mathrm{H}$ Barnett ${ }^{3}$ \\ 1. Professor of Medicine, Catholic University Leuven, and Head of Endocrinology, University Hospital Gasthuisberg; \\ 2. Diabetes Principal Medical Scientific Expert, Novartis Pharma AG; 3. Professor of Medicine, and Honorary Consultant Physician, \\ Division of Medical Sciences, University of Birmingham and Heart of England NHS Foundation Trust
}

DOI:10.17925/EE.2010.06.00.29

\begin{abstract}
For patients with diabetes, hypoglycaemia can present a number of risks ranging from mild to life-threatening in severity. Frequently occurring hypoglycaemia is associated with increased morbidity and mortality. Recent studies have found associations between intensive glycaemia treatment and an increased incidence of hypoglycaemia, prompting much discussion concerning the clinical significance of hypoglycaemia in the treatment of diabetes, especially considering that many hypoglycaemic episodes are subclinical and unrecognised. Hypoglycaemia has also been linked to an increased probability of developing dementia and, not surprisingly, poor quality of life as well. It may be helpful to try to prevent hypoglycaemic episodes through careful monitoring of patients with risk factors that predispose them to hypoglycaemia, while also selecting therapies that can minimise the incidence of hypoglycaemic episodes.
\end{abstract}

\section{Keywords}

Hypoglycaemia, type 2 diabetes, cardiovascular disease, insulin, sulphonylurea, metformin, thiazolidinediones, dipeptidyl peptidase-4 (DPP-4) inhibitors

Disclosure: Chantal Mathieu has received honoraria for lectures and advisory work from NovoNordisk, Eli Lilly, MSD, Novartis, sanofi-aventis, Servier, Medtronic and Lifescan Claudia Filozof is an employee of Novartis Pharma AG. Anthony H Barnett has received honoraria for lectures and advisory work from NovoNordisk, Eli Lilly, MSD, Novartis, BMS/AstraZeneca and sanofi-aventis.

Received: 25 September 2009 Accepted: 12 November 2009 Citation: European Endocrinology, 2010;6(1):29-34

Correspondence: Chantal Mathieu, LEGENDO, Katholieke Universitet, Leuven, UZ Gasthuisberg 0\&N, Herestraat 49, B-3000 Leuven, Belgium.

E: chantal.mathieu@uz.kuleuven.ac.be

Hypoglycaemia is a common problem for many patients with diabetes and the risks and consequences need to be considered when prescribing therapy. Mild episodes can cause unpleasant symptoms and disrupt daily activities, while severe hypoglycaemia can result in disorientation and unusual behaviour, and may be life-threatening. Frequent hypoglycaemia, as a complication of treatment, is associated with increased morbidity and mortality, making it a limiting factor in achieving adequate glycaemic control.1-6 In type 1 diabetes, hypoglycaemia has been recognised as a major hurdle for optimal glycaemic control for many years, with the Diabetes Control and Complications Trial (DCCT) clearly indicating an inverse relationship between lowering glycated haemoglobin $\left(\mathrm{HbA}_{1 \mathrm{c}}\right)$ and the occurrence of severe hypoglycaemia.? Therefore, the most important beneficial consequence of the introduction of insulin analogues in the treatment of patients with type 1 diabetes is not a major improvement in $\mathrm{HbA}_{1 \mathrm{C}}$, but rather a reduction in occurrence of hypoglycaemia. ${ }^{8}$

Although less frequent, many glucose-lowering therapies used in patients with type 2 diabetes can also cause hypoglycaemia, particularly sulphonylureas and insulin. Recent results from trials including the Action to Control Cardiovascular Risk in Diabetes (ACCORD) and the Veterans Affairs Diabetes Trial (VADT) have stimulated discussion of the potential long-term cardiovascular (CV) risk of hypoglycaemic events in patients with type 2 diabetes. The importance of avoiding hypoglycaemia is becoming increasingly considered in terms of diabetes care in both type 1 and 2 diabetes.
The true incidence of hypoglycaemia is unknown. Many episodes are subclinical and go unnoticed by both the physician and the patient. The frequency is likely to be significantly underestimated. The objective of this article is to discuss the impact and clinical implications of hypoglycaemia, with particular attention to people with type 2 diabetes.

\section{Definition of Hypoglycaemia}

Hypoglycaemia has been defined as a plasma glucose level of $<70 \mathrm{mg} / \mathrm{dl} \quad(<3.9 \mathrm{mmol} / \mathrm{l})$, as once levels go below this threshold, activation of the anti-insulin neuroendocrine counter-regulatory response normally occurs. ${ }^{1,2}$ However, the definition of hypoglycaemia used in clinical trials often varies between investigators and the threshold may range from 55 to $70 \mathrm{mg} / \mathrm{dl}$ (3 to $3.9 \mathrm{mmol} / \mathrm{l}$ ), leading to different estimations of its incidence. ${ }^{9,10}$ By contrast, more descriptive definitions have also been employed where the authors manually review and classify subjects into 'mild' (not requiring third-party help) or 'severe' (if the patient requires help). ${ }^{11}$ These different approaches to defining hypoglycaemia make it difficult to pinpoint its exact incidence.

\section{Frequency of Hypoglycaemia}

Although the true frequency of hypoglycaemia is hard to determine, in an analysis of 50,048 patients with type 2 diabetes, $4.1 \%$ suffered an episode of hypoglycaemia while on treatment with oral antidiabetes agents (OADs). In the same analysis, use of insulin was found to be a significant risk factor for hypoglycaemia, as was sulphonylurea (SU) 
Table 1: Frequency of Hypoglycaemia from Observational Studies in Outpatient Settings

\begin{tabular}{|c|c|c|c|}
\hline Study & Number & Frequency of Hyp & glycaemia \\
\hline & & Type 1 & Type 2 \\
\hline Jennings et al., 1989a & 219 & & $20 \%$ of those \\
\hline & & & taking SU \\
\hline Miller et al., 2000' & 1,055 & & $16 \%$ with OADs \\
\hline & & & $30 \%$ with insulin \\
\hline Shorr et al., $1997^{\circ}$ & 19,932 & & 1.23 severe \\
\hline & & & episodes/100 \\
\hline & & & person-years \\
\hline & & & with SUS \\
\hline & & & 2.76 severe \\
\hline & & & episodes/100 \\
\hline & & & person-years \\
\hline & & & with insulin \\
\hline Gurlek et al., 1999 & 165 & 0.15 severe & 0.15 severe \\
\hline & & episodes/patient/ & episodes/patient/ \\
\hline & & year with insulin & year with insulin \\
\hline Leese et al., $2003^{e}$ & 160 & $7.1 \%$ requiring & $7.3 \%$ requiring \\
\hline & & emergency & emergency \\
\hline & & treatment & treatment \\
\hline Donnelly et al., $2005^{f}$ & 267 & 42.9 per & 16.4 per \\
\hline & & patient/year & patient/year \\
\hline Henderson et al., $2003^{8}$ & 215 & & 0.28 severe \\
\hline & & & episodes/patient/ \\
\hline & & & year \\
\hline MacLeod et al., $1993^{h}$ & 600 & 1.7 episodes/ & 0.73 episodes/ \\
\hline & & patient/year & patient/year \\
\hline Hepburn et al., $1993^{\prime}$ & 172 & & $82.7 \%$ with insulin \\
\hline
\end{tabular}

$O A D S=$ oral antidiabetes agents; $S U=$ sulphonylurea

a. Jennings AM, Wilson RM, Ward JD, Symptomatic hypoglycaemia in NIDDM patients treated with oral hypoglycaemic agents, Diabetes Care, 1989:12:203-8.

b. Miller $C D$, Phillips $L S$, Ziemer $D C$, et al., Hypoglycaemia in patients with type 2 diabetes mellitus, Arch Intern Med, 2001;161:1653-9.

C. Shorr RI, Ray WA, Daugherty JR, et al., Incidence and risk factors for serious hypoglycaemia in older persons using insulin or sulfonylureas, Arch Intern Med 1997;157:1681-6.

d. Gurlek A, Erbas T, Gedik O, Frequency of severe hypoglycaemia in type 1 and type 2 diabetes during conventional insulin therapy, Exp Clin Endocrinol Diabetes, 1999;107:220-24.

e. Leese GP, Wang J, Broomhall J, et al., Frequency of severe hypoglycaemia requiring emergency treatment in type 1 and type 2 diabetes: a population-based study of health service resource use, Diabetes Care, 2003;26:1176-80.

f. Donnelly $L A$, Morris AD, Frier BM, et al., Frequency and predictors of hypoglycaemia in Type 1 and insulin-treated Type 2 diabetes: a population-based study, Diabet Med, 2005;22:749-55

g. Henderson JN, Allen KV, Deary IJ, et al., Hypoglycaemia in insulin-treated Type 2 diabetes: frequency, symptoms and impaired awareness, Diabet Med, 2003;20:1016-21.

h. MacLeod KM, Hepburn DA, Frier BM, Frequency and morbidity of severe hypoglycaemia in insulin-treated diabetic patients, Diabet Med, 1993;10:238-45.

i. Hepburn DA, MacLeod KM, Pell AC, et al., Frequency and symptoms of hypoglycaemia experienced by patients with type 2 diabetes treated with insulin, Diabet Med, 1993;10:231-7.

(adjusted odds ratio [OR] 3.73). ${ }^{11}$ By contrast, use of metformin was associated with a comparatively low risk (OR 1.42) - i.e. hypoglycaemia was nearly three times more common with SU than with metformin treatment in the absence of insulin. The recorded frequencies of hypoglycaemia in selected trials are listed in Table 1.

\section{Causes of Hypoglycaemia}

In patients with diabetes, causes of hypoglycaemia include missed or delayed food intake, a meal or snack that is too small, vigorous exercise without adequate carbohydrate compensation and alcohol consumption.' A major cause of hypoglycaemia in patients is the medicine used to control glycaemia. ${ }^{12}$ Relative or absolute insulin excess from therapy in combination with compromised physiological defences against falling plasma glucose concentrations can lead to hypoglycaemia., Insulin excess occurs because of an inadequate action profile of the drug when placed in the context of factors such as food intake, exercise, drug interactions and insulin clearance. Meanwhile, in type 1 and more advanced type 2 diabetes, there is impairment of all three physiological defences that would normally correct hypoglycaemia: there is no decrease in insulin levels, no increase in glucagon levels and the increase in epinephrine (adrenaline) levels may be attenuated, resulting in a higher hypoglycaemia risk with insulin use..$^{11,13,14}$

The potential of a specific OAD to cause hypoglycaemia is dependent on its mechanism of action. For example, SUs are insulin secretagogues and work by increasing insulin secretion regardless of blood glucose levels, effectively uncoupling the glucose sensitivity of pancreatic islet beta-cells and insulin secretion, thus increasing the risk of hypoglycaemia. ${ }^{15,16}$ By contrast, metformin does not directly stimulate insulin secretion and therefore the risk of hypoglycaemia is low."

\section{Impaired Awareness of Hypoglycaemia}

The initial symptoms of hypoglycaemia are largely triggered by increased levels of epinephrine (adrenaline), one of the counterregulatory responses normally associated with low blood glucose..$^{3,4}$ However, in many patients with type 1 and 2 diabetes of longer duration, counter-regulatory responses are blunted - a phenomenon known as hypoglycaemia-associated autonomic failure (HAAF) ${ }^{4,17,18}$ This syndrome not only involves reduced neuroendocrine counter-regulatory responses to lowered blood glucose, but also lowered glycaemic thresholds for activation of defences against hypoglycaemia. This latter can lead to hypoglycaemia unawareness, which is the inability to perceive the normal (early) warning symptoms of hypoglycaemia.

Studies suggest that even one prolonged, moderate episode of hypoglycaemia may be sufficient to significantly reduce counterregulatory responses. ${ }^{19,20}$ Without the warning signs, episodes become difficult to detect. Accordingly, the incidence of subclinical hypoglycaemia cannot be determined accurately, although episodes are common in insulin-treated patients. ${ }^{1}$ Indeed, the prevalence has been estimated as high as $19.5 \%$ in patients with type 1 diabetes. ${ }^{21}$ The major risk associated with episodes of subclinical hypoglycaemia is the potential for reduced awareness of the onset of a severe episode because the glycaemic threshold has been substantially lowered. In turn, a bout of recurrent episodes can lead to a vicious

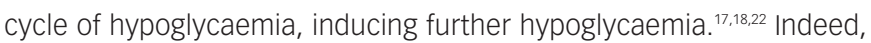
subclinical hypoglycaemia (not surprisingly) is associated with a sixfold increased risk of severe hypoglycaemia., ${ }^{21,23}$ In insulin-treated patients with type 2 diabetes, the prevalence of impaired awareness of hypoglycaemia has been estimated to be around $10 \%$. Impaired awareness of hypoglycaemia in this cohort was associated with a five-fold higher incidence of hypoglycaemia and a 17-fold higher incidence of severe episodes. ${ }^{10}$ On the other hand, studies suggest that several weeks of stringent avoidance of hypoglycaemia can reverse hypoglycaemia unawareness in the majority of patients. ${ }^{1,24-26}$

\section{Hypoglycaemia and Long-term Cardiovascular Morbidity and Mortality}

In addition to the immediate consequences of hypoglycaemia, episodes may also lead to other longer-term health consequences. Three recently published studies have examined the effects of intensive versus standard glycaemia treatment on CV risk in 
Hypoglycaemia in Type 2 Diabetes - Clinical Consequences and Impact on Treatment

Table 2: Comparison of the Three Trials of Intensive Glycaemic Control and Cardiovascular Disease Outcomes

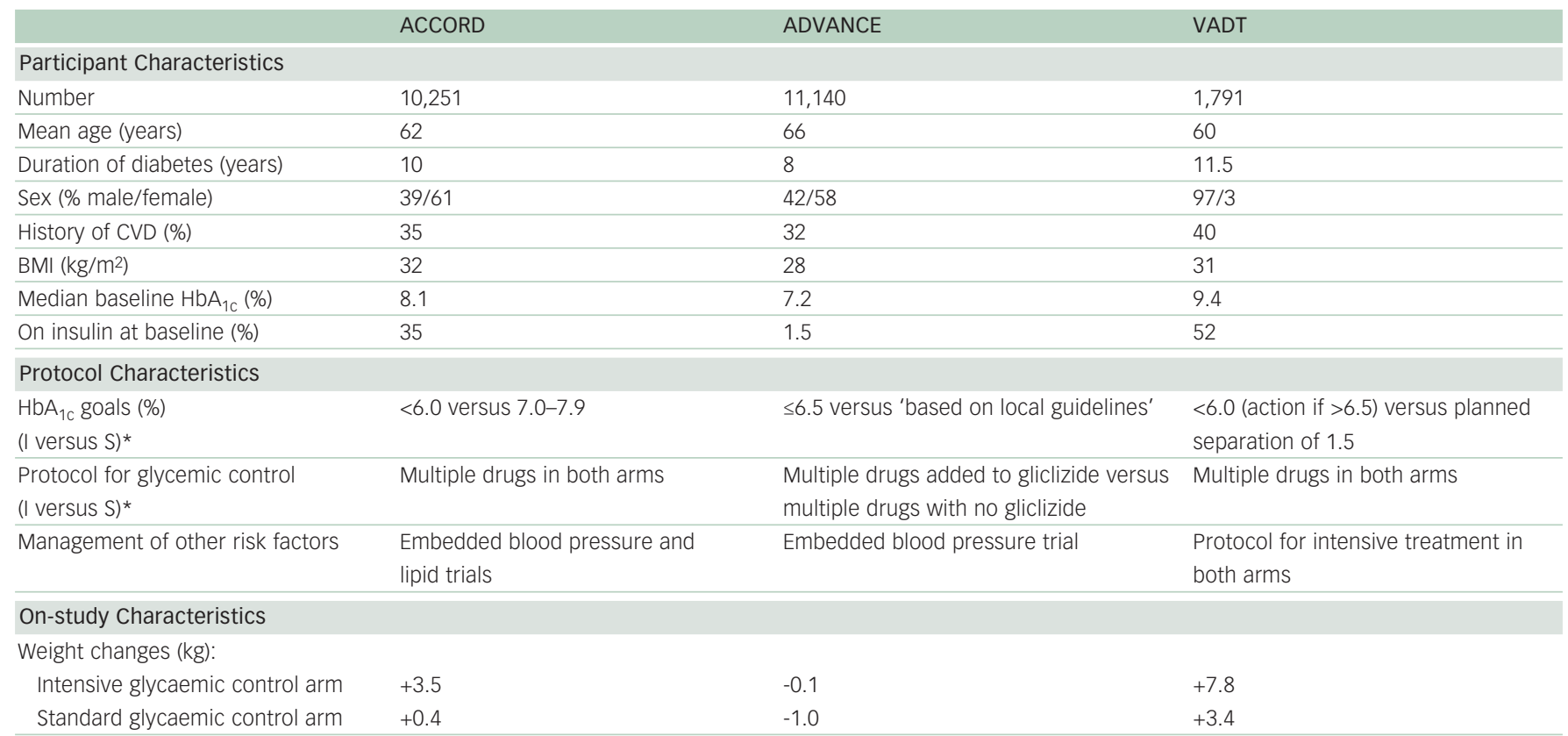

Severe hypoglycaemia (participants

with one or more episodes during

study) (\%):

Intensive glycaemic control arm 16

Standard glycaemic control arm 5.1

2.7
1.5

21.2

Outcomes

Definition of primary outcome

Non-fatal MI, non-fatal stroke, CVD death

HR for primary outcome $(95 \% \mathrm{Cl})$

$0.90(0.78-1.04)$

HR for mortality findings (95\% Cl) $1.22(1.01-1.46)$

Microvascular plus macrovascular (non-fatal MI, non-fatal stroke, CVD death) outcomes

0.9 (0.82-0.98); macrovascular 0.94 $(0.84-1.06)$

$0.93(0.83-1.06)$
9.9

Non-fatal MI, non-fatal stroke, CVD death, hospitalisation for heart failure, revascularisation $0.88(0.74-1.05)$

\section{$1.07(0.81-1.42)$}

ACCORD = Action to Control Cardiovascular Risk in Diabetes; ADVANCE = Action in Diabetes and Vascular Disease: Preterax and Diamicron Modified Release Controlled Evaluation; $V A D T=$ Veterans Affairs Diabetes Trial; $C V D=$ cardiovascular disease; I = intensive glycaemic control; $S=$ standard glycaemic control; $\mathrm{Cl}=$ confidence interval; $\mathrm{HR}=$ hazard ratio; $\mathrm{MI}=$ myocardial infarction. *Medication rates for ACCORD are for any use during the study.

patients with type 2 diabetes (see Table 2)..$^{27-29}$ In the three studies, the incidence of hypoglycaemia was significantly higher in the intensive therapy group. None found that near-normal glycaemic control (median $\mathrm{HbA}_{1 \mathrm{c}}$ of $6.4 \%$ at study end in the intensive group) significantly reduced the incidence of $\mathrm{CV}$ events within a 3.5-5-year time-frame..$^{27-29}$

By contrast, the ACCORD trial found that overall mortality was greater in the intensive therapy group. ${ }^{27-29}$ In 19 of the 41 unexpected excess deaths from $\mathrm{CV}$ causes in the ACCORD study, 'unexpected or presumed CV disease' was possibly related to, or precipitated by, severe hypoglycaemia. ${ }^{27-29}$ Interestingly, in the ACCORD study, previous occurrence of severe hypoglycaemia was one of the strongest predictors of a primary CV event regardless of the treatment arm. In addition, while hypoglycaemic events were more frequent in the intensive therapy group, this arm also saw a greater increase in weight gain of more than $10 \mathrm{~kg}$. These factors, as well as the differences in number of drugs and regimens, could have contributed to the final mortality rates.

In VADT, hypoglycaemia was one of the strongest predictors of CV death (hazard ratio [HR] 4.042, 95\% confidence interval [Cl] 1.449-11.276; $p=0.01$ ); other predictors were prior event and age..$^{30}$
$\mathrm{CV}$ risk may also be increased by recurrent subclinical hypoglycaemic episodes as a low blood glucose level stimulates sympathetic neural activation and catecholamine secretion, which can lead to arrhythmia and increased heart rate, blood pressure and overall workload of the heart. ${ }^{31}$ These haemodynamic changes can result in stress in the arterial wall and may contribute to destabilisation of atherosclerotic plaques, potentially precipitating an atherothrombotic event. Furthermore, acute hypoglycaemia causes physiological changes that affect the CV system and several haematological parameters, largely as a result of sympathoadrenal activation and counter-regulatory hormonal secretion. ${ }^{32}$

A study using healthy subjects found that antecedent hypoglycaemia led to impaired autonomic function, which could potentially contribute directly to mortality in diabetes and CV disease. ${ }^{33}$ By contrast, a study focusing on patients hospitalised with acute myocardial infarction (AMI) reported that hypoglycaemia only increased mortality when episodes occurred spontaneously without insulin treatment (18.4\% mortality rate in patients with hypoglycaemia versus $9.2 \%$ in those without). ${ }^{34}$ When hypoglycaemia was induced by insulin therapy, there was no significant difference in mortality between patients who suffered hypoglycaemia and those who did not (10.4 versus $10.2 \%$ ). Regardless of insulin treatment, the patients with hypoglycaemia were older and had more co-morbidities. ${ }^{34}$ Finally, the data also suggest that 
Table 3: Contributors to Increased Risk of Severe Hypoglycaemia

\begin{tabular}{l} 
Loss of Endogenous Insulin Secretion \\
Inability to reduce circulating insulin concentrations \\
Loss of signal to alpha-cells to increase glucagon secretion \\
\hline Possible loss of C-peptide or amylin effects \\
\hline Primary Failure of Hormones that Raise Blood Glucose Concentrations \\
Hypopituitarism \\
\hline Adrenal cortical failure \\
\hline Isolated growth hormone deficiency \\
\hline Defective Glucose Counter-regulation \\
Loss of glucagon response to hypoglycaemia \\
\hline Delayed onset of counter-regulatory response secondary to \\
antecedent hypoglycaemia \\
\hline Prolongation of Insulin Effect \\
Exogenous insulin injection \\
\hline Insulin secretagogues \\
\hline Renal impairment \\
\hline Hyperthyroidism \\
\hline High levels of insulin-binding antibodies \\
\hline Liver failure \\
\hline Exaggerated Mismatch Between Insulin and Nutrient Absorption \\
Primary gastrointestinal disease with malabsorption (coeliac disease) \\
\hline Delayed insulin administration \\
\hline Lifestyle Contributors to Individual Episodes of Severe Hypoglycaemia \\
\hline Use of
\end{tabular}

hypoglycaemia during hospitalisation for AMI was a marker for more severe illness and not necessarily a direct cause of it, providing some reassurance to physicians in their efforts for glycaemic control in patients. However, the question remains whether hypoglycaemia resulting from certain drugs is harmful to patients.

Analyses of ACCORD do not show any definitive relationship between the increased CV mortality in the intensive treatment arm and any combination of drugs used. However, the combination of an SU and metformin in ACCORD has previously been shown to be associated with a significantly higher rate of $\mathrm{CV}$ events and trends towards a higher rate of total mortality compared with SU or metformin monotherapy treatment in the United Kingdom Prospective Diabetes Study (UKPDS) ${ }^{35-37}$ Furthermore, a recent meta-analysis suggests that the combination of an SU plus metformin in patients with type 2 diabetes, while having no significant effects on either CV disease mortality or all-cause mortality alone, may increase the relative risk of the composite end-point of CV hospitalisation or mortality (fatal and non-fatal events). The authors presented several possible explanations for their observation, one of which is the tendency of SUs to cause hypoglycaemia, which may be further aggravated by the metformin causing decreased hepatic glucose production, possibly impairing the recovery from hypoglycaemia. However, the authors highlight the limitations that exist in their meta-analysis and stress the need for larger-scale studies to further characterise the results. ${ }^{38}$ Glyburide (glibenclamide), the most widely used SU, was linked to a $52 \%$ greater risk of experiencing at least one hypoglycaemic episode compared with other secretagogues and an 83\% greater risk compared with other SUs; ${ }^{39}$ however, it was not associated with a higher risk of $\mathrm{CV}$ events.
These studies suggest a possible association between hypoglycaemia and CV risk, leading to a need to find treatment options that have a lower risk of hypoglycaemic events to prevent potential long-term CV morbidity and mortality.

\section{Hypoglycaemia and Other Co-morbidities}

In addition to CV risk, hypoglycaemia may have an effect on risk of dementia in older patients with type 2 diabetes. ${ }^{40} \mathrm{~A}$ longitudinal cohort study was performed from 1980 to 2007 with 16,667 patients with a mean age of 65 years with type 2 diabetes. Severe hypoglycaemic episodes requiring hospitalisation significantly increased a patient's probability of developing dementia, with the risk increasing with more episodes. ${ }^{40}$ Further research is required to determine the association between minor episodes and risk of dementia. These results support the need for caution specifically in treating older patients with diabetes in order to limit the risk of dementia. They also illustrate the complexities connected with diabetes treatment, as any hypoglycaemia - caused by drugs or otherwise - may lead to a number of unknown consequences for the patient.

\section{Hypoglycaemia and Quality of Life}

Hypoglycaemia and its symptoms may cause fear in patients with diabetes and clinicians alike and can potentially lead to stress and an impaired quality of life for patients and clinical inertia within the medical community. Hypoglycaemic episodes have both short- and long-term consequences on health-related quality of life (HRQOL). The short-term consequences refer to the symptoms associated with the actual event. While hypoglycaemic episodes may be relatively harmless, episodes can result in unconsciousness or impaired cognition and thus situations may arise that can be detrimental for both the patient and surrounding individuals. ${ }^{41}$ The long-term consequences relate to changes in patient behaviour and their fear of future episodes, resulting in negative social and emotional states. ${ }^{41,42}$ Fear of hypoglycaemia is one of the most problematic long-term consequences. ${ }^{42}$ Patients who suffer hypoglycaemic episodes, even non-severe episodes, are more likely to experience anxiety and panic attacks that in turn can increase the number of episodes. ${ }^{41}$ An important clinical implication resulting from a fear of hypoglycaemia is reluctance by the patient and physician to intensify antidiabetes therapy that may lead to a negative impact on diabetes management, metabolic control and subsequent health outcomes. ${ }^{41,43}$ This clinical inertia, which has been summarised as "the failure to initiate or intensify therapy in a defined time among patients who have not attained clinical goals and whom intensification is likely to benefit", ${ }^{44}$ is a limiting factor in diabetes treatment. Moreover, in an attempt to avoid hypoglycaemia, some patients may alter treatment intensity and overeat to elevate blood glucose levels..$^{41}$ However, it has been shown that blood glucose awareness training can reduce fear in patients. ${ }^{42}$

\section{Individuals at High Risk and Clinical Implications of Hypoglycaemia}

Although many patients with diabetes are at risk of experiencing hypoglycaemic episodes, there are factors that predispose certain individuals to a greater risk. In older patients in particular, factors such as a restricted carbohydrate intake, renal and hepatic dysfunction and the effects of alcohol and medications in common use may increase the risk of hypoglycaemia (see Table 3).45,46 These aspects, in combination with SU or exogenous insulin therapy, make it more likely that a patient will experience hypoglycaemia. ${ }^{46}$ An inadequate glucagon response arising with long-standing diabetes, in particular in 
type 1 diabetes, puts patients with diabetes at greater risk as defences against hypoglycaemia are largely reduced. ${ }^{1}$ However, patients with long-standing type 2 diabetes with endogenous insulin deficiency who have been on insulin therapy for many years are also at increased risk of severe hypoglycaemia. ${ }^{47}$ Patients with a history of repetitive hypoglycaemia, in particular in combination with hypoglycaemia unawareness, are at particular risk of severe hypoglycaemic attacks. Furthermore, too aggressive glycaemic therapy resulting in lower glycaemic goals, lower $\mathrm{HbA}_{1 \mathrm{c}}$ levels or both can have also an effect on future hypoglycaemic episodes. ${ }^{1}$

Finally, special attention should be given to those patients in whom even mild hypoglycaemic attacks may have major consequences, e.g. people in certain occupations (those working at heights or with heavy machinery and occupational drivers) and people living alone. In these circumstances, great care should be taken to avoid hypoglycaemia altogether.

Given the potential severity of hypoglycaemic events and their effect on the health of patients, it is important to minimise their incidence. This can be accomplished by treating diabetes with drugs that have a lower risk of hypoglycaemia, such as metformin, thiazolidinediones and dipeptidyl peptidase-4 (DPP-4) inhibitors. The inhibition of the DPP-4 enzyme by this latter new class of drugs enhances the action of glucagon-like peptide-1 (GLP-1). As GLP-1 causes insulin secretion to increase only when glucose levels are elevated, hypoglycaemia is less likely to occur. ${ }^{48}$ In fact, the UK's National Institute for Health and Clinical Excellence (NICE) has recently recommended that DPP-4 inhibitors be used as a secondline therapy for patients with type 2 diabetes if the person is at significant risk of hypoglycaemia or its consequences. A recent meta-analysis of randomised clinical trials studied the role of DPP-4 inhibitors in the treatment of type 2 diabetes and found that DPP-4 inhibitors lowered $\mathrm{HbA}_{1 \mathrm{c}}$ with little or no weight gain or hypoglycaemia risk. However, further study is needed to determine the long-term safety profiles of this class of drug. ${ }^{49}$ Glitazones are another class of type 2 diabetes therapy that have been studied as a potential treatment with a low hypoglycaemia risk. These agents work by helping the body use its available amounts of insulin more effectively, thereby improving the patient's insulin sensitivity. As a result, glitazones are associated with a low incidence of hypoglycaemia. ${ }^{50}$ However, when either drug class is used in combination with SUs or insulin, the combination can still lead to blood glucose levels dropping below normal.

\section{Summary and Conclusions}

Hypoglycaemia and its potentially serious consequences are a major concern when managing patients with diabetes. ${ }^{22}$ Recurrent episodes of hypoglycaemia, especially severe events, can lead to poor treatment adherence in patients and a reluctance to intensify treatment by health professionals, as well as having a negative impact on patient quality of life. Hypoglycaemia was a predictor of CV events in both ACCORD and VADT (although a causal relationship was not definitely established) and might precipitate other morbidities, such as dementia. It would seem prudent to use therapies that are associated with a lower risk of hypoglycaemia (particularly in high-risk subjects) in an attempt to achieve optimal and early glycaemic control in patients with type 2 diabetes, while minimising the risk of adverse consequences.

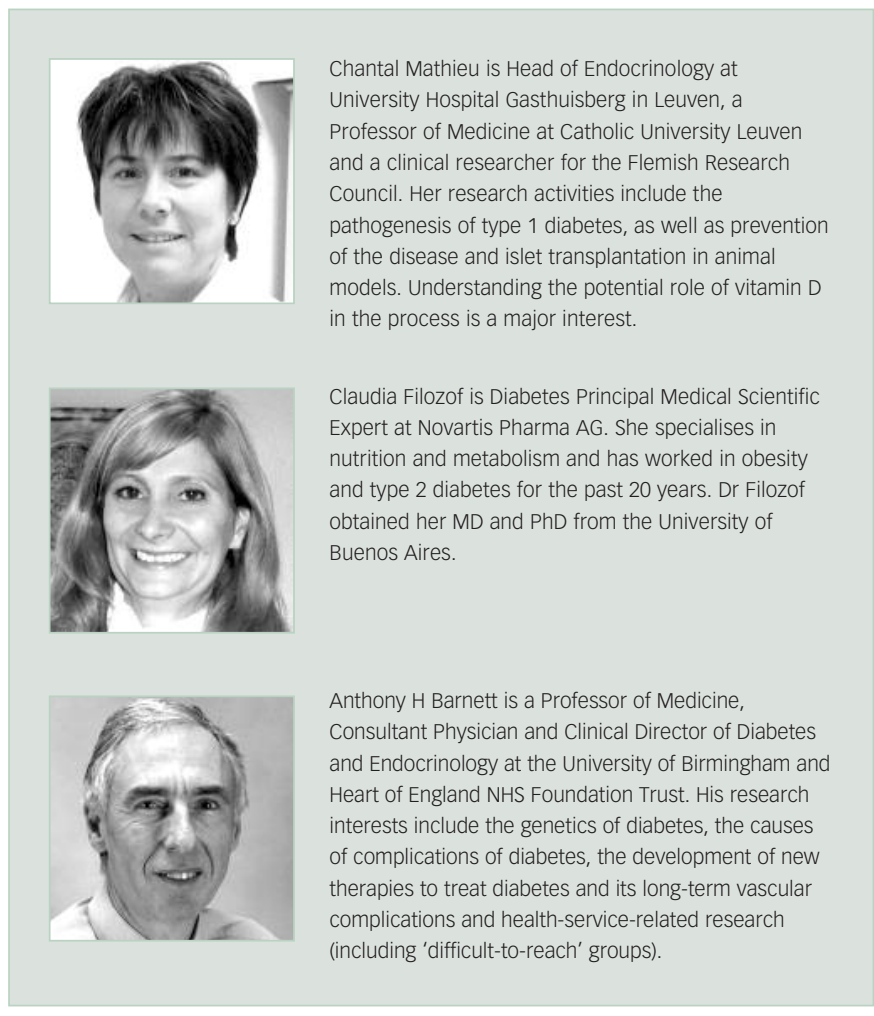

1. Workgroup on Hypoglycemia, American Diabetes Association, Defining and reporting hypoglycemia in diabetes: a report from the American Diabetes Association Workgroup on Hypoglycemia, Diabetes Care, 2005;28:1245-9.

2. Briscoe VJ, Davis SN, Hypoglycemia in type 1 and type 2 diabetes: Physiology, pathophysiology, and management, Clin Diabetes, 2006;24:115-21.

3. Cryer PE, Davis $\mathrm{SN}$, Shamoon $\mathrm{H}$, Hypoglycemia in diabetes, Diabetes Care, 2003;26:1902-12.

4. Cryer PE, Diverse causes of hypoglycemia-associated autonomic failure in diabetes, N Eng/ J Med, 2004;350: 2272-9.

5. United Kingdom Prospective Diabetes Study 24: a 6-year randomized, controlled trial comparing sulfonylurea, insulin, and metformin therapy in patients with newly diagnosed type 2 diabetes that could not be controlled with diet therapy. United Kingdom Prospective Diabetes Study Group, Ann Intern Med, 1998;128:165-75.

6. Turner R, Cull C, Holman R, United Kingdom Prospective Diabetes Study 17: a 9-year update of a randomized, controlled trial on the effect of improved metabolic control on complications in non-insulin-dependent diabetes mellitus, Ann Intern Med, 1996:124:136-45.

7. The effect of intensive treatment of diabetes on the development and progression of long-term complications in insulin-dependent diabetes mellitus. The Diabetes Control and Complications Trial Research Group, N Eng/ J Med, 1993;329:977-86.

8. Gough SC, A review of human and analogue insulin trials, Diabetes Res Clin Pract, 2007;77:1-15.

9. Fatourechi MM, Kudva YC, Murad MH, et al., Clinical review: Hypoglycemia with intensive insulin therapy: a systematic review and meta-analyses of randomized trials of continuous subcutaneous insulin infusion versus multiple daily injections, I Clin Endocrinol Metab, 2009;94: 729-40.

10. Schopman JE, Geddes J, Frier BM, Prevalence of impaired awareness of hypoglycaemia in insulin-treated type 2 diabetes and its effect on hypoglycaemia frequency, European Association for the Study of Diabetes (EASD), 2008; abstract 681.

11. Bodmer $\mathrm{M}$, Meier $\mathrm{C}$, Krahenbuhl $\mathrm{S}$, et al., Metformin, sulfonylureas, or other antidiabetes drugs and the risk of lactic acidosis or hypoglycemia: a nested case-control analysis, Diabetes Care, 2008;31:2086-91.

12. Fowler MJ, Hypoglycemia, Clin Diabetes, 2008;26:170-73.

13. Heller SR, Amiel SA, Mansell P, Effect of the fast-acting insulin analog lispro on the risk of nocturnal hypoglycemia during intensified insulin therapy. U.K. Lispro Study Group, Diabetes Care, 1999;22:1607-11.

14. Ratner RE, Hirsch IB, Neifing JL, et al., Less hypoglycemia with insulin glargine in intensive insulin therapy for type 1 diabetes. U.S. Study Group of Insulin Glargine in Type 1 Diabetes, Diabetes Care, 2000;23:639-43.

15. DeFronzo RA, Lilly lecture 1987. The triumvirate: betacell, muscle, liver. A collusion responsible for NIDDM, Diabetes, 1988;37:667-87.

16. Krentz AJ, Bailey $\mathrm{CJ}$, Oral antidiabetic agents: current role in type 2 diabetes mellitus, Drugs, 2005;65:385-411.

17. Dagogo-Jack SE, Craft S, Cryer PE, Hypoglycemiaassociated autonomic failure in insulin-dependent diabetes mellitus. Recent antecedent hypoglycemia reduces autonomic responses to, symptoms of, and defense against subsequent hypoglycemia, I Clin Invest, 1993;91:819-28. 
18. Segel SA, Paramore DS, Cryer PE, Hypoglycemiaassociated autonomic failure in advanced type 2 diabetes, Diabetes, 2002:51:724-33.

19. Davis SN, Tate D, Effects of morning hypoglycemia on neuroendocrine and metabolic responses to subsequent afternoon hypoglycemia in normal man, I Clin Endocrinol Metab, 2001;86:2043-50

20. Heller SR, Cryer PE, Reduced neuroendocrine and symptomatic responses to subsequent hypoglycemia after 1 episode of hypoglycemia in nondiabetic humans, Diabetes, 1991;40:223-6.

21. Geddes J, Schopman JE, Zammitt NN, et al., Prevalence of impaired awareness of hypoglycaemia in adults with Type 1 diabetes, Diabet Med, 2008;25:501-4.

22. Cryer PE, Mechanisms of hypoglycemia-associated autonomic failure and its component syndromes in diabetes, Diabetes, 2005:54:3592-3601.

23. Gold AE, MacLeod KM, Frier BM, Frequency of severe hypoglycemia in patients with type I diabetes with impaired awareness of hypoglycemia, Diabetes Care, 1994;17:697-703.

24. Dagogo-Jack S, Rattarasarn C, Cryer PE, Reversal of hypoglycemia unawareness, but not defective glucose counterregulation, in IDDM, Diabetes, 1994;43:1426-34.

25. Fanelli C, Pampanelli S, Epifano L, et al., Long-term recovery from unawareness, deficient counterregulation and lack of cognitive dysfunction during hypoglycaemia following institution of rational, intensive insulin therapy in IDDM, Diabetologia, 1994;37:1265-76.

26. Fanelli $\mathrm{CG}$, Epifano L, Rambotti AM, et al., Meticulous prevention of hypoglycemia normalizes the glycemic thresholds and magnitude of most of neuroendocrine responses to, symptoms of, and cognitive function during hypoglycemia in intensively treated patients with short-term IDDM, Diabetes, 1993;42:1683-9.

27. Duckworth W, Abraira C, Moritz T, et al., Glucose contro and vascular complications in veterans with type 2 diabetes, N Eng/ I Med, 2009;360:129-39.

28. Gerstein $\mathrm{HC}$, Miller ME, Byington RP, et al., Effects of intensive glucose lowering in type 2 diabetes, $N$ Engl J Med, 2008;358:2545-59.
29. Patel A, MacMahon S, Chalmers J, et al., Intensive blood glucose control and vascular outcomes in patients with type 2 diabetes, N Eng/ J Med, 2008:358:2560-72.

30. Abraira C, Moritz TE, Reaven P, et al., Glycemic control and cardiovascular outcomes - the VA Diabetes Trial, Presented at: American Diabetes Association (ADA) 68th Annual Meeting and Scientific Sessions, San Francisco, 6-10 June 2008

31. Hoogwerf BJ, Does intensive therapy of type 2 diabetes help or harm? Seeking accord on ACCORD, Cleve Clin J Med, 2008;75:729-37.

32. Wright RJ, Frier BM, Vascular disease and diabetes: is hypoglycaemia an aggravating factor?, Diabetes Metab Res Rev, 2008;24:353-63.

33. Adler GK, Bonyhay I, Failing $\mathrm{H}$, et al., Antecedent hypoglycemia impairs autonomic cardiovascular function: implications for rigorous glycemic control Diabetes, 2009;58:360-66.

34. Kosiborod M, Inzucchi SE, Goyal A, et al., Relationship between spontaneous and iatrogenic hypoglycemia and mortality in patients hospitalized with acute myocardial infarction, JAMA, 2009;301:1556-64.

35. UKPDS 28: a randomized trial of efficacy of early addition of metformin in sulfonylurea-treated type 2 diabetes. U.K. Prospective Diabetes Study Group, Diabetes Care, 1998;21:87-92

36. Nathan DM, Some answers, more controversy, from UKPDS. United Kingdom Prospective Diabetes Study, Lancet, 1998;352:832-3

37. Turner RC, Cull CA, Frighi V, et al., Glycemic control with diet, sulfonylurea, metformin, or insulin in patients with type 2 diabetes mellitus: progressive requirement fo multiple therapies (UKPDS 49). UK Prospective Diabetes Study (UKPDS) Group, JAMA, 1999;281:2005-12.

38. Rao AD, Kuhadiya N, Reynolds $K$, et al., Is the combination of sulfonylureas and metformin associated with an increased risk of cardiovascular disease or allcause mortality?: a meta-analysis of observational studies, Diabetes Care, 2008:31:1672-8.

39. Gangji AS, Cukierman T, Gerstein HC, et al., A systematic review and meta-analysis of hypoglycemia and cardiovascular events: a comparison of glyburide with other secretagogues and with insulin, Diabetes Care, 2007;30:389-94

40. Whitmer RA, Karter AJ, Yaffe K, et al., Hypoglycemic episodes and risk of dementia in older patients with type 2 diabetes mellitus, JAMA, 2009:301:1565-72

41. Levy AR, Christensen TL, Johnson JA, Utility values for symptomatic non-severe hypoglycaemia elicited from persons with and without diabetes in Canada and the United Kingdom, Health Qual Life Outcomes, 2008;6:73

42. Wild D, von Maltzahn R, Brohan E, et al., A critical review of the literature on fear of hypoglycemia in diabetes: Implications for diabetes management and patient education, Patient Educ Couns, 2007;68:10-15.

43. Nordfeldt $\mathrm{S}$, Jonsson $\mathrm{D}$, Short-term effects of severe hypoglycaemia in children and adolescents with type 1 diabetes. A cost-of-illness study, Acta Paediatr, 2001:90: 137-42.

44. Phillips LS, Branch WT, Cook CB, et al., Clinical inertia, Ann Intern Med, 2001:135:825-34.

45. Epidemiology of severe hypoglycemia in the diabetes control and complications trial. The DCCT Research Group, Am J Med, 1991;90:450-59.

46. United Kingdom Prospective Diabetes Study (UKPDS). 13: Relative efficacy of randomly allocated diet sulphonylurea, insulin, or metformin in patients with newly diagnosed non-insulin dependent diabetes followed for three years, BMJ, 1995;310:83-8.

47. Dokken BB, The parodox of glycaemic control and cardiovascular complications: sorting out the data, Diab Spect, 2008;21(3):150-52.

48. Meade LT, DPP-4 inhibitors and the quest for HDA1C control, US Pharm, 2007;32:HS-24-HS-HS-32.

49. Monami M, lacomelli I, Marchionni N, et al., Dipeptydil peptidase- 4 inhibitors in type 2 diabetes: A metaanalysis of randomized clinical trials, Nutr Metab Cardiovasc Dis, 2009 (Epub ahead of print)

50. Rosenstock J, Baron MA, Dejager S, et al., Comparison of vildagliptin and rosiglitazone monotherapy in patients with type 2 diabetes: a 24-week, double-blind randomized trial, Diabetes care, 2007:30:217-23. 